\section{Regulating type III interferon}

Type III interferons (IFN- $\lambda 1-I F N-\lambda 3$ ) exert highly circumscribed antiviral effects dictated mainly by the limited expression of their receptor, IFNLR1, on mucosal epithelium. In PLoS Biology, Robek and colleagues reveal the epigenetic mechanisms that control this restricted pattern of IFNLR1 expression. The amount of methylation of the IFNLR1 promoter inversely correlates with the expression of this receptor. Conversely, as gene expression is positively regulated by acetylation, knockdown or chemical inhibition of histone deacetylases increases the acetylation and expression of IFNLR1. Cells that are normally insensitive to IFN- $\lambda$ can be made responsive by inhibition of histone deacetylases and can thereby be rendered resistant to viral infection. Acetylation of the IFNLR1 promoter then allows access of the transcription factor NF-Y, which controls IFNLR1 expression. Selectively controlling expression of IFNLR1 through the use of smallmolecule drugs may be useful in sensitizing cells to the antiviral effects of type III interferons.

PLoS Biol (7 January 2014) doi:10.1371/journal.pbio. 1001758

\section{Killing bystander T cells}

Infection with human immunodeficiency virus (HIV) results in the depletion of $\mathrm{CD}^{+} \mathrm{T}$ cells in patients with AIDS. In Science, Monroe et al. describe how quiescent $\mathrm{T}$ cells are killed by abortive infection with HIV. Quiescent CD $4^{+}$T cells can be infected with HIV but fail to support viral production or the establishment of latent provirus. Instead, these cells accumulate incomplete viral cDNAs, which are then recognized by IFI16, a cytosolic DNA sensor that is induced by interferon- $\gamma$. IFI16 forms active inflammasomes that cleave caspase- 1 and initiates pyroptosis, contributing to HIV pathogenesis. Knockdown of IFI16 or inhibition of HIV reverse transcriptase rescues quiescent $\mathrm{T}$ cells from this form of cell death induced by abortive HIV infection. Thus, HIV coopts the host-cell innate response. Such killing of bystander T cells might account for much of the loss of $\mathrm{CD} 4^{+} \mathrm{T}$ cells observed in patients with AIDS.

$L A D$ Science 343, 428-432 (2014)

\section{Heart macrophages}

Fate-mapping studies have demonstrated the prenatal origin of macrophages in many tissues and their ability to undergo population expansion and be maintained in situ. In Immunity, Mann and colleagues characterize macrophage populations in the myocardium at steady state and after cardiac stress. Four subsets of cardiac-resident macrophages exist separately from the blood and are renewed through in situ proliferation at steady state. Most adult cardiac macrophages are established embryonically, with a substantial contribution from the yolk sac. In a model of cardiac infarction, macrophage numbers increase through both the in situ proliferation of resident macrophages and the recruitment of blood Ly6Chi monocytes, which differentiate into all four macrophage subsets to different degrees. Transcriptional analysis shows that monocyte-derived macrophages coordinate cardiac inflammation, while both blood-derived macrophages and embryoderived macrophages have a role in antigen sampling.

Immunity 40, 91-104 (2014)

Written by Laurie A. Dempsey, Zoltan Fehervari \& Ioana Visan

\section{Ascl2 for $\mathrm{T}_{\mathrm{FH}}$ cells}

The development of follicular helper T cells ( $\mathrm{T}_{\mathrm{FH}}$ cells) is initiated when T cells migrate into the $\mathrm{B}$ cell follicle as they upregulate expression of the chemokine receptor CXCR5. In Nature, Dong and colleagues show that the helix-loop-helix factor Ascl2 initiates $\mathrm{T}_{\mathrm{FH}}$ cell development. Ascl2 expression in $\mathrm{T}_{\mathrm{FH}}$ cells is dependent on Wnt agonists but is independent of transcription factors known to regulate $\mathrm{T}_{\mathrm{FH}}$ cell development, such as Bcl-6, Batf and STAT5. Ascl2 induces Cxcr5 expression by directly binding to E-boxes in the Cxcr5 promoter and represses genes related to the $\mathrm{T}_{\mathrm{H}} 1, \mathrm{~T}_{\mathrm{H}} 2$ and $\mathrm{T}_{\mathrm{H}} 17$ subsets of helper $\mathrm{T}$ cells through a transcriptional profile distinct from that of Maf, Batf and IRF4, which are required for $\mathrm{T}_{\mathrm{FH}}$ differentiation. Acute deletion of Ascl 2 in vivo leads to total impairment of $\mathrm{T}_{\mathrm{FH}}$ cell development and germinal center response in mice. In addition, the E-protein inhibitor Id 3 inhibits Ascl 2 function and the generation of $\mathrm{T}_{\mathrm{FH}}$ cells, which suggests that $\mathrm{E}$-box proteins control $\mathrm{T}_{\mathrm{FH}}$ differentiation.

Nature (19 January 2014) doi:10.1038/nature12910

\section{Limiting NKT cells}

Invariant natural killer T cells (iNKT cells) rapidly produce cytokines in response to CD1d-presented lipid antigens. In Cell, An et al. report that the commensal gut bacterium Bacteroides fragilis limits NKT cell numbers early in life and this results in protection against colitis in adults. $B$. fragilis expresses an enzyme, SPT, required for sphingolipid biosynthesis. Wild-type bacteria synthesize $\alpha$-galactosylceramides that can be loaded onto CD1d molecules; however, these molecules fail to stimulate $i$ NKT cells and function to competitively inhibit other lipid molecules for presentation by CD1d. $B$. fragilis colonization in neonatal mice limits $I_{\text {NKT }}$ cell proliferation and diminishes the total number of iNKT cells. However germ-free mice or those monocolonized by $B$. fragilis SPT mutants have more $i$ NKT cells in the gut and thus develop heightened cytokine responses when challenged with agonist antigens of $i N K T$ cells. Why $i N K T$ cell numbers are particularly sensitive within an early developmental window remains unknown, but these findings suggest ways of intervening with iNKT cell responses.

Cell 156, 123-133 (2014)

\section{Selecting dominance}

The precise extent to which thymic positive selection influences the subsequent immundominance hierachy of T cell antigen receptors (TCRs) is unclear. In eLife, Allen and colleagues investigate this by generating a mouse line (gp250 SC) that expresses a single $\mathrm{CD} 4^{+} \mathrm{T}$ cell-selecting ligand in the thymus that consists of the self gp250 peptide presented by $\mathrm{I}^{\mathrm{k}} \mathrm{k}$. 'Normal' mice primed by the nominal foreign antigen MCC generate a consistent and discrete TCR immundominance hierachy, and the gp250 peptide is able to positively select the most immunodominant TCR $\left(\mathrm{V}_{\alpha} 11^{+} \mathrm{V}_{\beta} 3^{+}\right)$in these mice. The gp250 SC mice produce a diverse TCR repertoire but have a much higher frequency of $\mathrm{CD} 4^{+}$cells able to recognize MCC-derived peptides and have a TCR dominance hierachy similar to that of 'normal' mice primed with MCC. Furthermore, amino acid residues in complementarity-determining region 3 of the gp250 SC TCR show skewing toward recognition of MCC. These data demonstrate that a positively selecting ligand can directly determine the nature of the immunodominant $\mathrm{T}$ cell response.

eLife 110, 20176-20181 (2014) 